\title{
MicroRNA-124 inhibits the proliferation of C6 glioma cells by targeting Smad4
}

\author{
ZECHUAN ZHANG $^{1 *}$, QIAOYUN GONG ${ }^{2 *}$, MEIYING LI $^{1}$, JINYING XU $^{1}$, \\ YANGYANG ZHENG ${ }^{1}$, PENGFEI GE ${ }^{3,4}$ and GUANGFAN CHI ${ }^{1}$
}

\footnotetext{
${ }^{1}$ The Key Laboratory of Pathobiology, Ministry of Education, College of Basic Medical Sciences, Jilin University; ${ }^{2}$ Eye Center, The Second Hospital of Jilin University; ${ }^{3}$ Department of Neurosurgery, First Hospital of Jilin University; ${ }^{4}$ Research Center of Neuroscience, First Hospital of Jilin University, Changchun, Jilin 130021, P.R. China
}

Received August 6, 2016; Accepted July 20, 2017

DOI: $10.3892 /$ ijmm.2017.3088

\begin{abstract}
MicroRNA-124 (miR-124) has been shown to be downregulated in glioma; however, its biological functions in glioma are not yet fully understood. The aim of this study was to examine the Smad4-dependent effects of miR-124 on C6 glioma cell proliferation. In this study, the level of miR-124 was found to be enhanced in C6 cells upon transfection with miR-124 mimics, and the mechanisms of action of miR-124 in C6 cells were investigated by reverse transcriptase-quantitative polymerase chain reaction, MTT assay, western blot analysis and luciferase reporter assays in vitro. The results revealed that miR-124 expression was significantly lower in the C6 cells than in either normal rat brain tissue or astrocytes. Upon the overexpression of miR-124, the proliferation of the C6 cells decreased and Smad4 expression was significantly suppressed. Smad4 was identified as a direct target of miR-124 through luciferase reporter assays. Furthermore, miR-124 was found to modulate signal transducer and activator of transcription 3 (Stat3) by downregulating Smad4 expression. Using small interfering RNA targeting Smad4 mRNA, we also confirmed that miR-124 downregulated c-Myc by modulating Smad4 expression. In addition, caspase- 3 expression was induced by miR-124 overexpression, but not via Smad4 downregulation. On the whole, our results demonstrate that miR-124 upregulation inhibits the growth of C6 glioma cells by targeting Smad 4 directly. These findings may be clinically useful for the development of therapeutic strategies directed toward miR-124 function in patients with glioma.
\end{abstract}

Correspondence to: Professor Guangfan Chi, The Key Laboratory of Pathobiology, Ministry of Education, College of Basic Medical Sciences, Jilin University, 126 Xinmin Street, Changchun, Jilin 130021, P.R. China

E-mail: guangfan130@jlu.edu.cn

${ }^{*}$ Co-first authorship

Key words: microRNA-124, glioma, Smad4, proliferation, Stat3, c-Myc

\section{Introduction}

Gliomas account for $29 \%$ of all primary brain tumors and are classified by the World Health Organization (WHO) into 4 grades of malignancy (1). Grade IV astrocytoma [glioblastoma multiforme (GBM)] is the most aggressive form of brain tumor in adults and is characterized by rapid cell proliferation, immunosuppressive capability and high malignancy (2). GBM is typically resistant to surgery, radiation, chemotherapy and drug treatment. Thus, there is an urgent need to elucidate the underlying molecular mechanisms involved in the genesis and progression of glioma, and to develop effective therapies.

Transforming growth factor $\beta$ (TGF- $\beta$ ) has been demonstrated to function as an oncogenic factor in high-grade glioma, and an increased TGF- $\beta$ expression can enhance glioma outgrowth and prevent glioma-initiating cell (GIC) differentiation $(3,4)$. The phenomenon has also been shown to be associated with a high expression of leukemia-inhibitory factor (LIF) induced by the TGF- $\beta /$ Smad signaling pathway (5). In general, TGF- $\beta$ functions by binding to type I and II TGF- $\beta$ receptors on the cell membrane. Upon binding TGF- $\beta$, type II receptors phosphorylate and activate type I receptors, which then propagate the signal by phosphorylating the receptor-regulated Smads (R-Smads), Smad2 and Smad3. These R-Smad members can form a complex with Smad4, which subsequently translocates to the nucleus and governs target gene expression (6). Therefore, these signal transducing proteins of the TGF- $\beta$ pathway are potential therapeutic targets for effectively treating patients with glioma.

MicroRNAs (miRNAs or miRs) comprise a class of small non-coding RNAs, which regulate gene expression during both normal and neoplastic development. miRNAs are generated by RNA polymerase II as longer precursor RNAs in the nucleus and contain hairpins. Subsequently, precursor RNAs are transported to the cytoplasm and are processed into 21-23 nucleotide-long RNA molecules by the RNase III enzymes Drosha and Dicer $(7,8)$. Mature miRNAs regulate gene expression by inhibiting mRNA translation or inducing mRNA degradation after binding to the complementary sequences in the 3'-untranslated regions (3'-UTRs) of target mRNAs. Emerging data have indicated that miRNAs are involved in tumor proliferation and apoptosis and can function either as oncogenes or 
tumor suppressor genes $(9,10)$. A number of miRNAs have been identified, including some that are involved in the malignant progression of glioma $(11,12)$. In particular, miR-124 plays a crucial role in neurogenesis and stimulates neuronal differentiation. The expression level of miR-124 is high in the central nervous system, but is low or even absent in high-grade glioma $(13,14)$. The loss of miR-124 expression has been shown to enhance stem cell-like traits and to increase the invasive ability of glioma cells (15). Furthermore, miR-124 overexpression in GBM has been shown to result in decreased migration and invasion $(16,17)$. In addition, miR-124 has been shown to inhibit the signal transducer and activator of transcription 3 (Stat3)-signaling pathway to promote $\mathrm{T}$ cell-mediated immune clearance of glioma (18). Therefore, miR-124 may serve as an effective therapeutic target if its functional network is clarified.

Using target-prediction software programs (TargetScan and Segal Lab), we noted that putative pairing regions for miR-124-3p exist within the 3'-UTR of rat and human Smad4 mRNA. In this study, our primary aim was to explore the associations between miR-124 and Smad4, as well as their effects on glioma. Establishing the mechanism whereby miR-124 regulates glioma progression may provide fundamental supportive evidence for novel clinical therapies for glioma.

\section{Materials and methods}

Animals. All animals were supplied by the Experimental Animal Center of Jilin University (Changchun, China). All procedures involving animals were approved by the Ethics Committee of Jilin University and conformed to regulatory standards.

Cell culture. Rat C6 glioma (Cell Bank of the Chinese Academy of Science in Shanghai, China) were cultured in DMEM/F12 supplemented with 10\% fetal bovine serum (FBS; both from Gibco, Grand Island, NY, USA), $100 \mathrm{U} / \mathrm{ml}$ penicillin and $100 \mu \mathrm{g} / \mathrm{ml}$ streptomycin (Genview, Calimesa, CA, USA), at $37^{\circ} \mathrm{C}$ in a humidified atmosphere with $5 \% \mathrm{CO}_{2}$. The cells were passaged at a ratio of 1:3 when they became confluent.

Astrocytes were isolated from the cortices of neonatal rats (1-3 days old, $n=6$ ) as previously described by Schwartz and Wilson with some modifications (19). Following the removal of all meninges carefully from the brain surface, the rat cortex tissue was dissected from brain and completely triturated using fire-polished Pasteur pipettes. The destroyed tissues were then treated with $0.25 \%$ Trypsin/EDTA for $30 \mathrm{~min}$, and passed through $40 \mu \mathrm{m}$ nylon mesh and centrifuged to collect the enzymatically dissociated cells. The collected cells were suspended in the above-mentioned complete culture medium, and plated in $25 \mathrm{~T}$ flasks and cultured for almost 2 weeks; the medium was replaced every 2 days.

When the cells were confluent, the flasks were shaken at $250 \mathrm{rpm}$ overnight at $37^{\circ} \mathrm{C}$. After replacing with fresh medium, the cells were treated with cytosine arabinoside (5 $\mu \mathrm{M}$; Sigma, St. Louis, MO, USA) for $48 \mathrm{~h}$. Th cells were then subcultured to the next passage. The percentage of GFAP-expressing cells at passage 1 in these cultures was found to be $>95 \%$.

Cell transfection. To perform cell transfection, the C6 cells were plated at $2 \times 10^{5}$ cells/well. After $24 \mathrm{~h}$, the cells were transfected with the miR-124 mimic, negative control (NC), small interfering RNA against Smad4 (siR-Smad4), or an siR-NC (50 nM; GenePharma, Shanghai, China), using Lipofectamine 2000 (Invitrogen, Carlsbad, CA, USA), after which they were incubated at $37^{\circ} \mathrm{C}$ for $5 \mathrm{~h}$. Subsequently, the supernatant was replaced with fresh medium, and the cells were cultured for a further $24 \mathrm{~h}$.

RNA extraction and RT-qPCR. Total RNA was extracted using TRIzol reagent (Invitrogen). To analyze miRNA-124 expression, total RNA was polyadenylated and reverse transcribed using the All-in-One ${ }^{\mathrm{TM}}$ miRNA First-Strand cDNA Synthesis kit (GeneCopoeia, Rockville, MD, USA). The level of miRNA-124 (RmiRQP0074; GeneCopoeia) produced in the cells and tissues was detected using the All-in-OneTM miRNA RT-qPCR Detection kit (GeneCopoeia). Specific primers (GeneCopoeia) were used to amplify miR-124-3p, and RnU6 was used as a normalization control for miRNA expression.

To analyze mRNA expression, cDNA was synthesized using the GoScript ${ }^{\mathrm{TM}}$ Reverse Transcription system (Promega, Madison, WI, USA). Real-time PCR reactions were performed using SYBR Premix Dimer-Eraser (Takara, Dalian, China). GAPDH was used as a normalization control for mRNA expression. The qPCR primer sequences were as follows: GAPDH forward, 5'-AGACAGCCGCATCTTCTTGT-3' and reverse, 5'-CTTGCCGTGGGTAGAGTCAT-3'; Smad4 forward, 5'-CCA TCAGTCTGTCTGCTGCT-3' and reverse, 5'-TGATGCTCT GTCTCGGGTAG-3'; LIF forward, 5'-GTGCCAATGCCCTC TTTATT-3' and reverse, 5'-TGGTCTTCTCTGTCCCA TTG-3'. Relative quantification numbers were calculated using the $2^{-\Delta \Delta \mathrm{Ct}}$ method, which is based on the ratio of gene expression between an experimental group and a control group.

Immunofluorescence staining. The C6 cells were fixed in $4 \%$ paraformaldehyde. Normal goat serum was used to block nonspecific binding. A rabbit anti-Smad2/3 antibody (1:800; \#8685; Cell Signaling Technology, Inc., Danvers, MA, USA) and a mouse anti-Smad4 antibody (1:100; sc-7966; Santa Cruz Biotechnology, Santa Cruz, CA, USA) were used as a primary antibody. Following an overnight incubation with the primary antibody at $4^{\circ} \mathrm{C}$, the cells were further incubated with goat anti-rabbit Alexa Fluor 594 (1:200; \#111-585-144) and goat anti-mouse Alexa Fluor 488 (1:200; \#111-545-146)-conjugated secondary antibodies (Jackson ImmunoResearch Inc., West Grove, PA, USA) for $1 \mathrm{~h}$ at room temperature. Nuclei were stained with Hoechst 33342 (Invitrogen).

Western blot analysis. Total protein samples were collected from the C6 cells after lysing them in RIPA cell lysis buffer containing PMSF and phosphorylase inhibitors (Beijing Dingguo Changsheng Biotechnology Co., Ltd., Beijing, China). The lysates were centrifuged at $12,000 \mathrm{rpm}$ for $15 \mathrm{~min}$ at $4^{\circ} \mathrm{C}$. Protein concentrations were determined using the Bicinchoninic Acid Protein Assay kit (Beyotime Institute of Biotechnology, Haimen, China). The proteins were denatured for $5 \mathrm{~min}$. The proteins were then concentrated in 5\% sodium dodecyl sulfate-polyacrylamide gel electrophoresis (SDS-PAGE) stacking gels, separated on $10 \%$ SDS-PAGE gels, and transferred onto PVDF membranes (Roche, Mannheim, Germany). The membranes were then blocked in 5\% non-fat milk and 
incubated with antibodies against Smad4 (1:1,000; \#38454), Stat3 (1:1,000; \#12640), phosphorylated-Stat3 (p-Stat3; 1:2,000; \#9145), c-Myc (1:1,000; \#5605), cleaved caspase-3 $(1: 1,000 ; \# 9664)$ and GAPDH $(1: 1,000 ; \# 5174)$ (all from Cell Signaling Technology, Inc.). The PVDF membranes were then washed with Tris-buffered saline containing 1\% Tween-20 and probed with the secondary antibody peroxidase-conjugated goat anti-rabbit IgG (H+L) (1:5,000; \#111453; Beijing Dingguo Changsheng Biotechnology Co., Ltd.) for $2 \mathrm{~h}$ at room temperature. The signal was detected by chemiluminescence using the ECL-Plus detection system (Amersham Pharmacia Biotech, Arlington Heights, IL, USA). The densitometric intensities of protein bands were semi-quantified using Bandscan 5.0 (Glyko Biomedical, Novato, CA, USA) software and values were normalized to those of GAPDH for each sample.

Cell proliferation assay. The viability of the C6 cells transfected with miR-124 mimics, NC, siR-Smad4, or siR-NC was assessed at 3 time points (days 1,2, and 3 ) after seeding $2 \times 10^{3}$ transfected cells/well into 96-well culture plates. Cell proliferation was measured using the MTT Assay kit (Beyotime Institute of Biotechnology). According to the manufacturer's instructions, the absorbance was measured at $450 \mathrm{~nm}$ using a Infinite M200 PRO NanoQuant (Tecan, Männedorf, Switzerland). Three independent experiments were performed for each group.

Enzyme-linked immunosorbent assays (ELISAs). The C6 cells were cultured in DMEM/F-12 supplemented with 10\% FBS until they reached $70 \%$ confluence. Subsequently, the C6 cells were transfected with the miR-124 mimic, NC, siR-Smad4 or siR-NC (50 nM final concentration), incubated at $37^{\circ} \mathrm{C}$ for $5 \mathrm{~h}$, and the supernatant was then replaced with fresh medium and the cells were grown for a further $24 \mathrm{~h}$ before the medium was collected and filter-sterilized. LIF secretion was quantified using an LIF ELISA kit (Elabscience, Wuhan, China), according to the manufacturer's instructions.

Target gene bioinformatic prediction and luciferase reporter assay. The target site of rno-miR-124-3p within the 3'-UTR region of SMAD4 $\mathrm{mRNA}$ was predicted using the target-prediction software programs (http://www.targetscan.org/vert_71/) and Segal Lab online microRNA prediction tool (https:/genie. weizmann.ac.il/pubs/mir07/mir07_prediction.html).

For luciferase reporter assay, the 3'-UTR of Smad4 was cut from the PUC57-Smad4 vector (Promega) and inserted into the psiCHECK-2 vector (Promega), using the XboI (New England BioLabs, Ipswich, MA, USA) and NotI (New England BioLabs) restriction enzyme sites, and the resulting vector was designated as psiCHECK-Smad4. The predicted miR-124 binding site of the Smad4 3'-UTR was mutated and then inserted into the psiCHECK-2 vector, which was designated as psiCHECK-Smad4-mut. Constructs were verified by sequencing. 293 cells (Cell Bank of the Chinese Academy of Sciences) were cultured in 48-well plates in DMEM/F-12 supplemented with $10 \%$ FBS without antibiotics until they reached $\sim 80 \%$ confluency. The cells were then co-transfected with rno-miR-124-3p or miR-NC $(50 \mathrm{nM})$ and psiCHECK-Smad4 or psiCHECK-Smad4-mut (100 pg), using Lipofectamine 2000. Following transfection for $5 \mathrm{~h}$, the medium was replaced with fresh mediaum containing
$10 \%$ FBS. Following a 48-h transfection, light emission was measured. The luciferase assay was conducted using the Dual-Glo Luciferase Assay system (Promega), following the manufacturer's instructions. Light emission was measured using a GloMax ${ }^{\circledR} 96$ Microplate Luminometer (Promega). The ratios of Renilla versus firefly luciferase signals served as a measurement of reporter activity normalized for the transfection efficiency.

Statistical analysis. Experimental data are presented as the means \pm standard deviation. All statistical analyses were performed and all graphs were prepared using GraphPad Prism 6 statistical software (GraphPad Software, Inc., La Jolla, CA, USA). Differences were considered statistically significant with a value of $\mathrm{P}<0.05$.

\section{Results}

miR-124 expression is low in C6 cells and the overexpression of miR-124 inhibits cell proliferation. To explore the functional roles of miR-124 in the proliferation and apoptosis of glioma cells, miR-124 expression was analyzed in C6 cells, non-tumor rat brain tissues and normal rat astrocytes by RT-qPCR. The level of miR-124 expression in the C6 cells was significantly lower than that in the non-tumor rat brain tissues and astrocytes (Fig. 1A). To overexpress miR-124, the C6 cells were transfected with miR-124 mimics for $48 \mathrm{~h}$, and the control cells were transfected separately with negative control (NC) for $48 \mathrm{~h}$. The level of miR-124 distinctly increased in the miR-124 mimic-transfected group, compared to that observed in the NC group (Fig. 1B). In addition, we observed that C6 cell growth decreased following miR-124 overexpression (Fig. 1C). MTT assays were conducted to confirm the changes in the proliferation of the C6 cells following the overexpression of miR-124. The results demonstrated that miR-124 upregulation significantly inhibited the proliferation of C6 cells for up to 3 days (Fig. 1D). These results implied that low miR-124 expression may play a crucial role in GBM progression.

Smad4 is a direct target of $m i R-124$. The TGF- $\beta /$ Smad pathway is considered a potential target for the clinical treatment of glioma. As shown in this study, the Smad2, Smad3, and Smad4 proteins were strongly expressed in the $\mathrm{C} 6$ cells under normal culture conditions, indicating that the TGF- $\beta$ /Smad pathway was activated (Fig. 2A). Using target-prediction software programs (TargetScan and Segal Lab), the 1921bp-1926bp of nucleotides sequence within 3'-UTR of the rat Smad4 mRNA (NM_019275.3) was identified as a theoretical seed site of miR-124 (Fig. 2B). Hence, miR-124 may affect downstream Smad4 effector functions in the cytoplasm of C6 cells by regulating Smad4 expression. RT-qPCR analysis revealed that the Smad4 mRNA level was significantly decreased in the C6 cells upon the overexpression of miR-124 (Fig. 2C). The transient overexpression of miR-124 in the C6 cells also downregulated Smad4 at the protein level (Fig. 2D). The protein levels of $\mathrm{Smad} 2 / 3$ were measured to determine whether miR-124 regulates Smad2/3 expression. However, no marked differences in their expression were observed (Fig. 2D). These results implied that miR-124 regulated Smad4 expression without influencing Smad2/3 expression. 

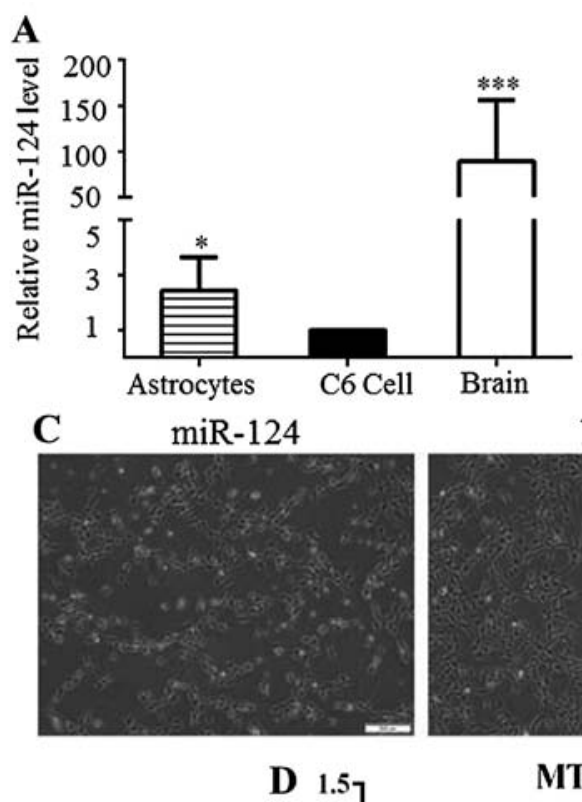
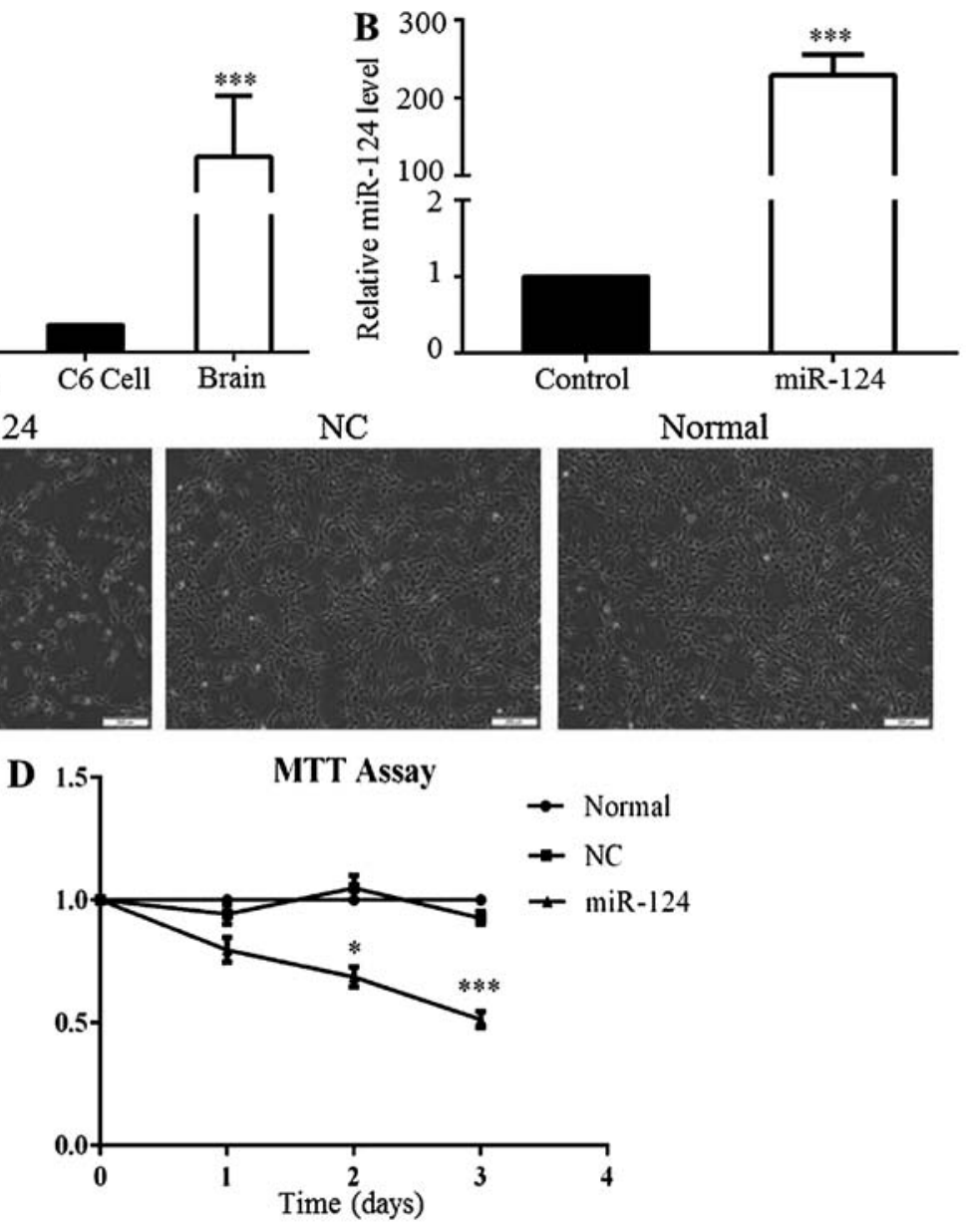

Figure 1. MicroRNA-124 (miR-124) expression in C6 cells inhibits C6 proliferation in vitro. miR-124 expression was measured by RT-qPCR and normalized to RnU6 expression. miR-124 overexpression was induced by transfecting C6 cells with a miR-124 mimic. (A) Relative expression levels of miR-124 in C6 cells, astrocytes and non-tumor brain tissues. The level of miR-124 in C6 cells was clearly lower than that in non-tumor brain tissues ( $\left.{ }^{* * *} \mathrm{P}<0.001\right)$. In addition, the miR-124 expression level in C6 cells was lower than that in astrocytes $\left({ }^{*} \mathrm{P}<0.05\right)$. (B) Transfection with a miR-124 mimic significantly increased the expression of miR-124 in C6 cells $\left.{ }^{* * *} \mathrm{P}<0.001\right)$. (C) The number of cells significantly declined in miR-124 group compared to the NC group and untreated group as observed by phase-contrast microscopy (200 mm). (D) MTT assays were performed using C6 cells transfected with the miR-124 mimic or negative control (NC). Compared to the NC group, $\mathrm{C} 6$ cell proliferation was clearly decreased in cells transfected with the miR-124 mimic $\left({ }^{*} \mathrm{P}<0.05,{ }^{* * *} \mathrm{P}<0.001\right)$.

To confirm that Smad4 is indeed directly targeted by miR-124, we examined whether miR-124 recognized the 3'-UTR of Smad4 mRNA by performing Dual-Luciferase reporter assays. The 3'-UTR of Smad4 was inserted into the the psiCHECK-2 vector, after which several constructs were sequenced. Smad4-mut was created as a control vector. The data indicated that miR-124 overexpression significantly inhibited the luciferase activity of psiCHECK-smad4, but not that of psiCHECK-smad4-mut (Fig. 2E). These results confirmed that miR-124 targets the 3'-UTR of Smad4 to directly suppress its expression.

Downregulation of Smad4 inhibits C6 cell proliferation. The complete pathological significance of Smad4 in glioma remains unclear. Thus, in this study, to explore the potential role of Smad4 in C6 cells, Smad4 was knocked down by transfection with siR-Smad4 or siR-NC. AT $24 \mathrm{~h}$ after transfection, Smad4 expression was significantly decreased in the siR-Smad4 group, compared to that in the siR-NC group (Fig. 3A and B). As with miR-124 overexpression, $\mathrm{Smad} 4$ downregulation also restricted the proliferation of the C6 cells (Fig. 3C). The results from MTT assay also confirmed that $\mathrm{C} 6$ cell proliferation was inhibited by the knockdown of Smad4 (Fig. 3D). We can thus conclude that Smad4 downregulation inhibited C6 cell proliferation, and thus the inhibitory effects of miR-124 on C6 cell growth interfered with Smad4 expression.

miR-124 reduces Stat3 expression by directly targeting Stat3 and through the modulation of Smad4 expression. Based on the TargetScan results (www.targetscan.com) and previous research (18), miR-124 binds to the 3'-UTR of Stat3. Theoretically, miR-124 can inhibit Stat 3 expression and thus overcome Stat3-mediated downstream suppression in GBM. Western blot analysis was performed to confirm the effects of miR-124 on the Jak/Stat pathway. In agreement with a previous study (18), Stat 3 and p-Stat 3 production was inhibited at the protein level by miR-124 overexpression (Fig. 4A). Previously, Peñuelas et al demonstrated that the TGF- $\beta / S m a d$ complex can activate the Jak/Stat Pathway via the induction of LIF in patient-derived GICs (5). Thus, in this study, to determine whether differences in Smad4 expression affect Stat3 expression, the C6 cells were transfected with siR-Smad4 or siR-NC, after which western blot analysis was conducted. The results revealed that reducing Smad4 expression significantly 
A
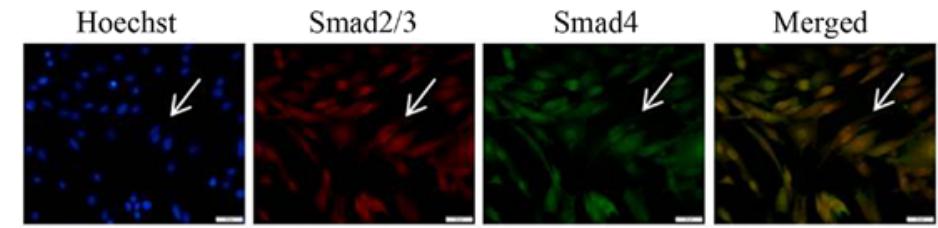

B

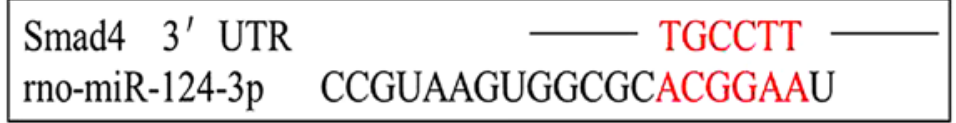

C

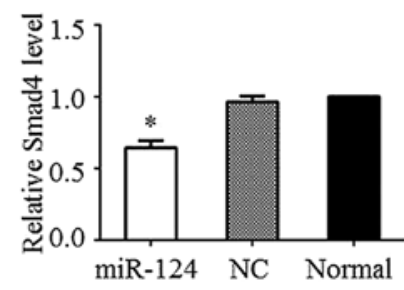

D
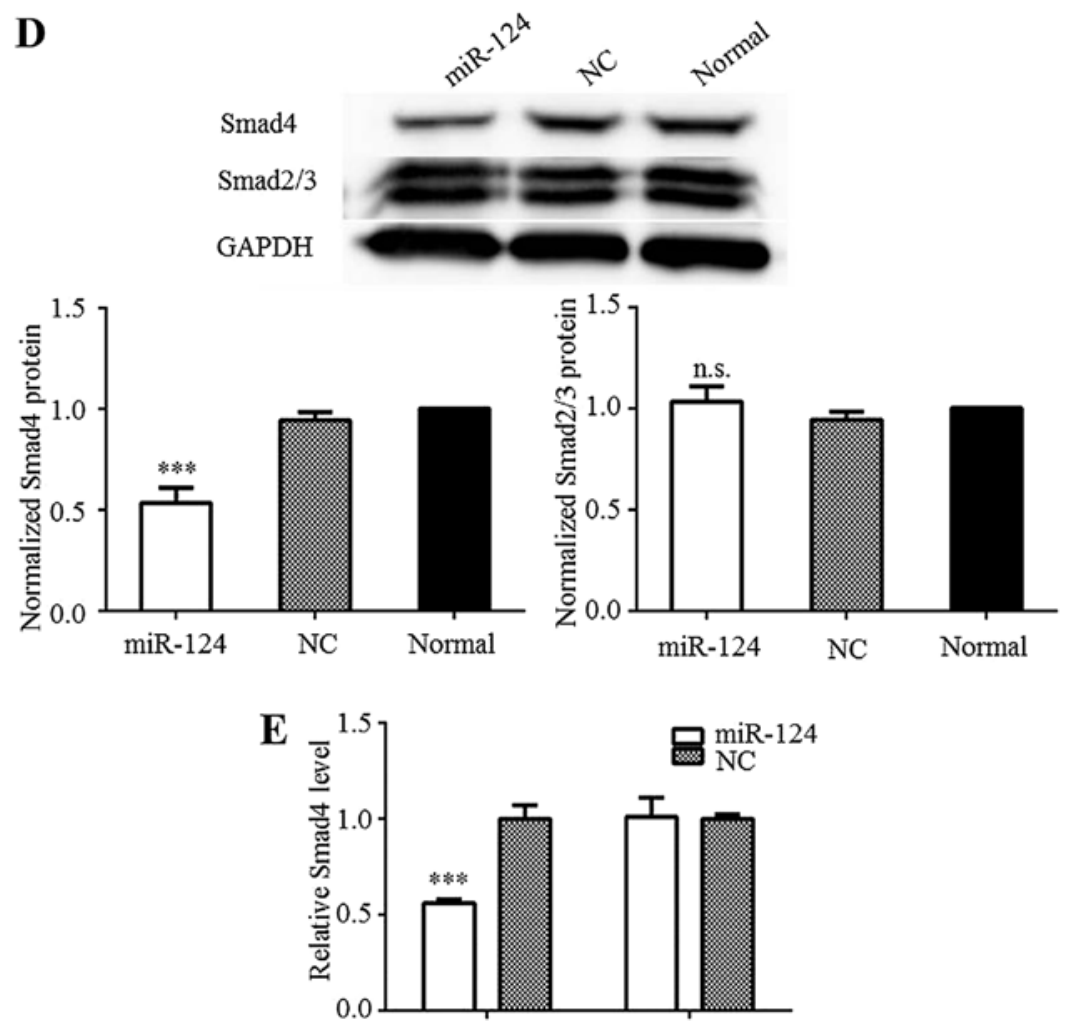

Figure 2. Smad4 directly targets microRNA-124 (miR-124) and inhibits its expression in C6 cells. C6 cells were transfected with the miR-124 mimic or negative control (NC) for $24 \mathrm{~h}$. Smad4 mRNA levels were measured by RT-qPCR. Total protein was extracted and subjected to western blot analysis. (A) Expression of Smad2/3 and Smad4 was confirmed in C6 cells by immunofluorescence. (B) Predicted miR-124 target sequences within the 3'-untranslated region (3'-UTR) of Smad4 mRNA. (C) Smad4 mRNA expression decreased significantly after miR-124 upregulation ("P<0.05). (D) Protein levels of Smad4 and Smad2/3 were detected using appropriate antibodies, and GAPDH protein expression was measured as an internal control. miR-124 inhibited Smad4 expression $(* * * * 0.001)$, but not Smad2/3 expression. (E) Luciferase activity of psiCHECK-smad4 3'-UTR. miR-124 distinctly suppressed the activity of the Smad4 3'-UTR in contrast to Smad4-mut $\left({ }^{* * *} \mathrm{P}<0.001\right)$, confirming Smad4 as a target of miR-124. n.s., not significant.

decreased Stat 3 expression (Fig. 4B). In addition, to determine whether Stat 3 expression was also regulated by miR-124 through LIF induction, the level of LIF was detected by RT-qPCR and ELISA. The RT-qPCR results demonstrated that siR-Smad4 reduced the mRNA level of LIF (Fig. 4C), but the ELISA results revealed that no significant change occurred at the protein level (Fig. 4D) These results may be due to the basal level of LIF secretion which is low in C6 cells. Taken together, these results indicate that Stat 3 expression can be directly suppressed by miR-124 and by Smad4-dependent suppression in C6 cells, but not through LIF induction.

miR-124 inhibits the proliferation of C6 cells and decreases $c-$ Myc expression. Zhu et al reported that TGF- $\beta$ activates both Smad-dependent and Smad-independent pathways, recruiting Smad4 to the Smad-binding element (SBE) of the c-Myc promoter, thereby promoting cell proliferation (20). In this study, c-Myc expression significantly decreased in the presence of 

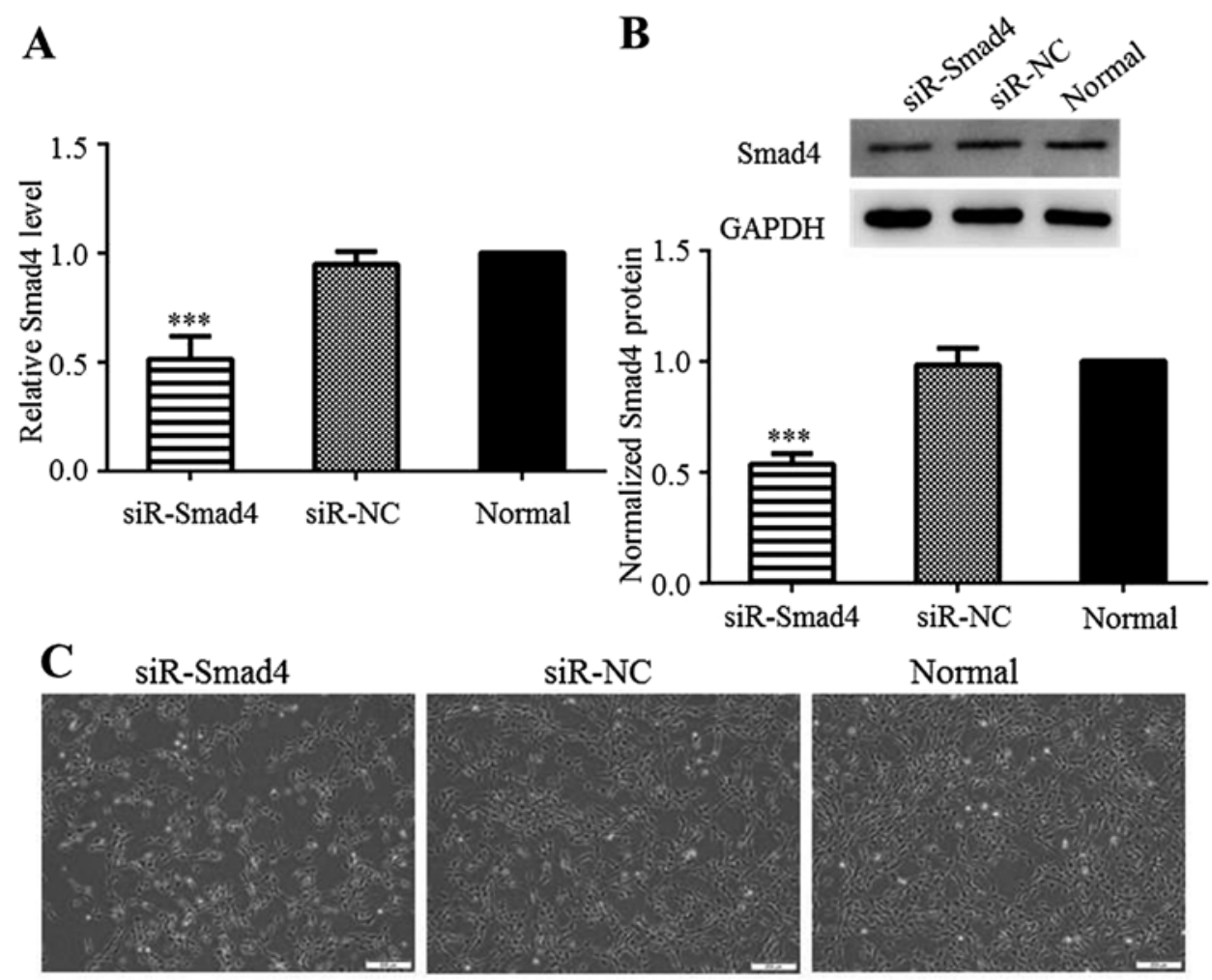

D

MTT Assay

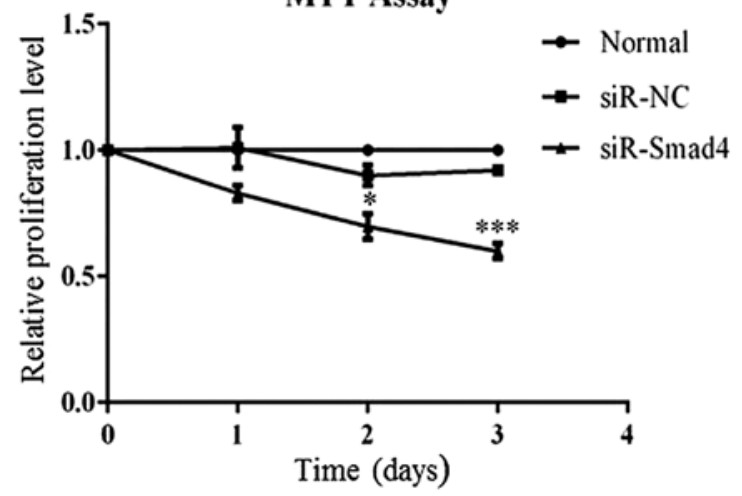

Figure 3. Knockdown of Smad4 inhibits the proliferation of glioma cells. C6 cells were transfected with small interfering RNA against Smad4 (siR-Smad4) or siR-negative control (NC) for $24 \mathrm{~h}$. (A) Smad4 mRNA expression was decreased in C6 cells following transfection with siR-Smad4, relative to that observed following transfection with the siR-NC $\left({ }^{* * *} \mathrm{P}<0.001\right)$. (B) Smad4 protein expression was detected using appropriate antibodies and was normalized to GAPDH expression. Smad4 was downregulated after transfection with siR-Smad4 ( $\left.{ }^{* * *} \mathrm{P}<0.001\right)$. (C) The number of cells significantly declined in siR-Smad4 treated cells compared to siR-NC and untreated cells as observed by phase-contrast microscopy (200 mm). (D) MTT assays were conducted in C6 cells following transfection with siR-Smad4 or siR-NC, revealing that cell proliferation was inhibited in C6 cells with low Smad4 expression $\left({ }^{*} \mathrm{P}<0.05\right.$, $\left.{ }^{* * *} \mathrm{P}<0.001\right)$.

miR-124 mimics and siR-Smad4 in C6 cells (Fig. 5A). Cleaved caspase-3 was detected by western blot anaysis to determine whether apoptosis occurs in the C6 cells after miR-124 overexpression. Cleaved caspase-3 was only distinctly expressed in the C6 cells after miR-124 overexpression (Fig. 5B). Based on these results, we suggest that miR-124 may influence the apoptosis of C6 glioma cells, but not through Smad4 modulation. Furthermore, the overexpression of miR-124 suppressed Smad4 production, leading to c-Myc downregulation, which inhibited the proliferation of $\mathrm{C} 6$ cells.

\section{Discussion}

In the present study, we confirmed the following: i) miR-124 expression was significantly downregulated in C6 glioma cells compared to in normal rat brain tissue and astrocytes, which was consistent with a previous report (15); ii) restoring miR-124 expression inhibited C6 cell proliferation, which correlated with Smad4 downregulation; and iii) Smad4 is a novel direct target gene of miR-124 that controls c-Myc expression in $\mathrm{C} 6$ cells.

Glioma is the most aggressive tumor type of the nervous system, and recurrence is high following therapy $(21,22)$. To date, many aspects of the molecular mechanisms of glioma tumorigenesis and progression have been investigated. miR-124 has been classified as a tumor suppressor in several types of human cancers (23-25). A number of studies have generated data indicating that miR-124 expression is significantly suppressed in glioma cells compared with normal brain tissue, and miR-124 expression levels negatively correlate with the 
A
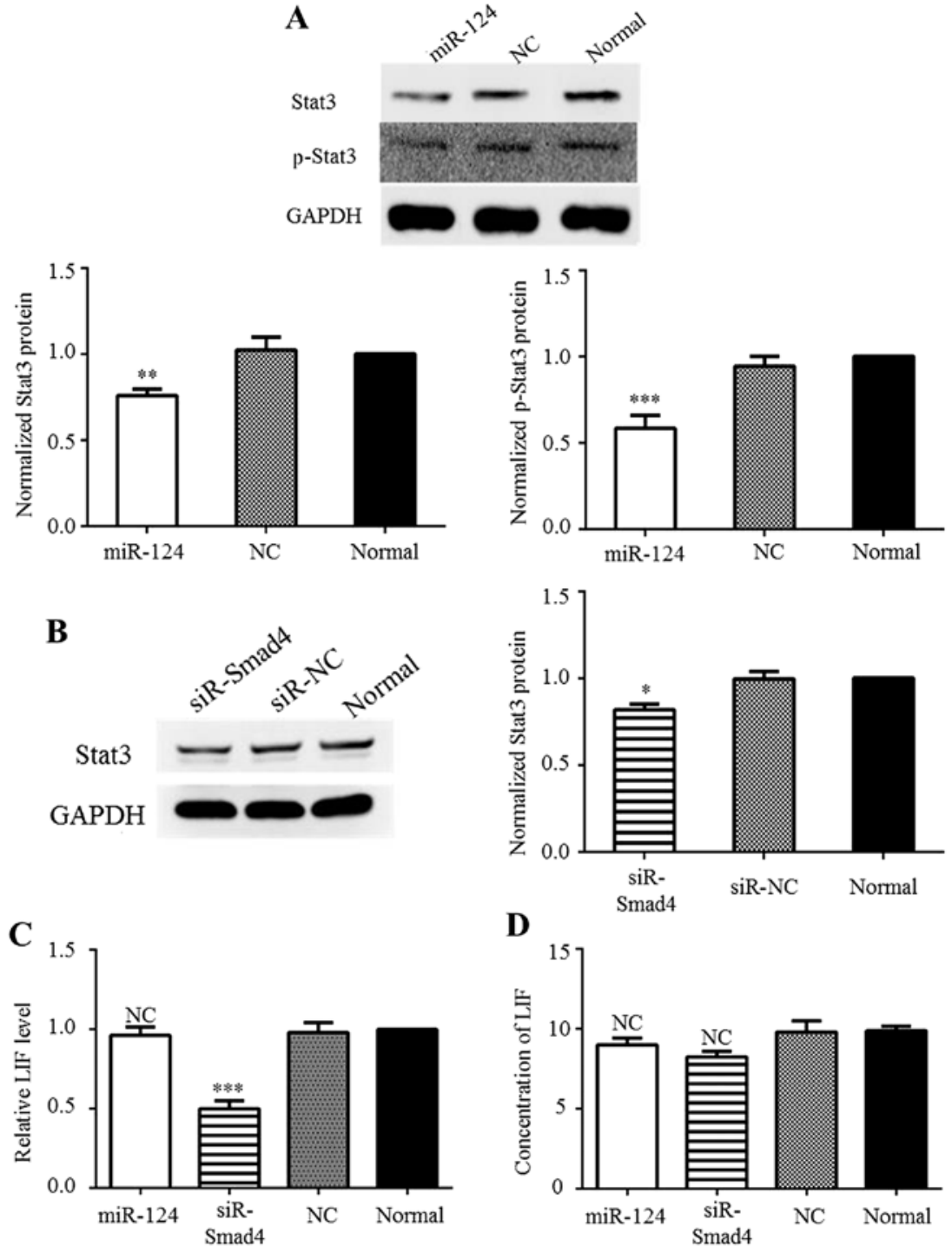

Figure 4. Signal transducer and activator of transcription 3 (Stat3) is regulated directly by microRNA-124 (miR-124) and indirectly by Smad4. C6 cells were treated with a miR-124 mimic or small interfering RNA against Smad4 (siR-Smad4) for $24 \mathrm{~h}$, after which cell proteins were extracted for western blot analysis. GAPDH was used as an internal control. The normal group was untreated. (A) Stat3 or phosphorylated-Stat3 (p-Stat3) expression in C6 cells overexpressing miR-124 was detected using appropriate antibodies. miR-124 upregulation repressed the protein-expression levels of Stat $3\left({ }^{* *} \mathrm{P}<0.01\right)$ and $\mathrm{p}-\mathrm{Stat} 3\left({ }^{* * *} \mathrm{P}<0.001\right)$. (B) Stat 3 expression was detectable in C6 cells with low Smad4 expression, and inhibiting Smad4 expression decreased Stat 3 production ("P<0.05). (C) Compared to that observed in the siR-Smad4 and negative control (NC) group, leukemia-inhibitory factor (LIF) mRNA expression was downregulated in the presence of siR-Smad4 $\left({ }^{* * *} \mathrm{P}<0.001\right)$, although no significant difference $(\mathrm{P}>0.05)$ was observed between the miR-124 and NC groups. (D) No significant change in the LIF protein level was observed following transfection with the miR-124 mimic or siR-Smad4 $(\mathrm{P}>0.05)$.
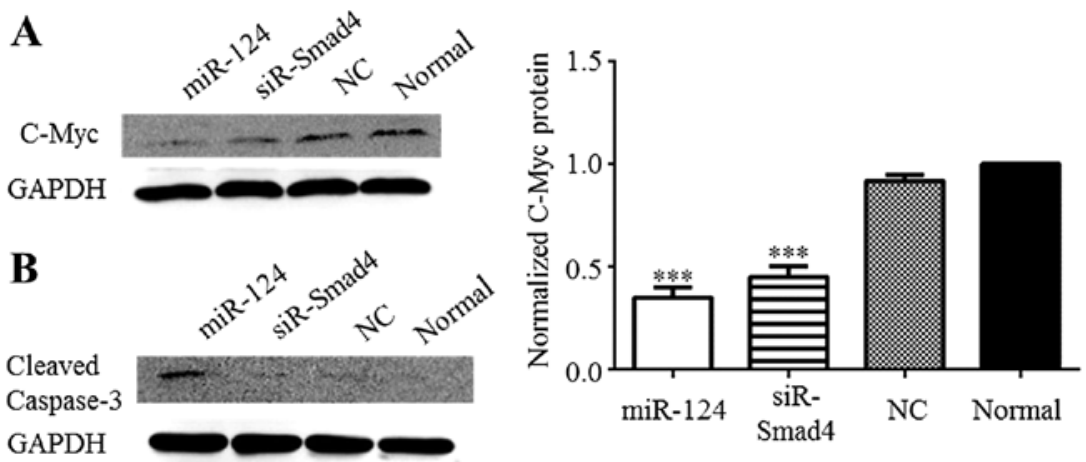

Figure 5. MicroRNA-124 (miR-124) inhibits C6 cell proliferation by repressing c-Myc expression. C6 cells were transfected separately with a miR-124 mimic or small interfering RNA against Smad4 (siR-Smad4). Expression of c-Myc and the apoptosis-related molecule cleaved caspase-3 were measured by western blot analysis, with GAPDH expression serving as an internal control. (A) The blot bands showed approximately a 1-fold decrease in c-Myc expression in C6 cells after miR-124 overexpression, or low-level Smad4 expression compared to the negative control (NC) group $\left.{ }^{(* * *} \mathrm{P}<0.001\right)$. (B) Cleaved caspase-3 expression in the presence of variable miR-124 expression showed that miR-124 promoted C6 cell apoptosis to inhibit their growth. 
pathological grade of glioma $(15,16,18,26-28)$. Several genes that control the biological behavior of glioma cells, such as self-renewal (proliferation), migration and invasion are direct targets of miR-124, including Stat3, IQGAP1, ROCK1, SOS1, SLC16A1, and CDK4 (18,26-30). These results indicate that in glioma cells, miR-124 functions through the post-transcriptional downregulation of multiple target genes. As with other miRNAs, miR-124 has numerous potential target genes and the underlying molecular mechanisms whereby miR-124 controls glioma cell proliferation are not limited to those target genes mentioned above.

TGF- $\beta$ is a well-known growth factor and is generally considered to function as a tumor suppressor. However, emerging evidence has indicated that in some tumor types, specifically in high-grade glioma, TGF- $\beta$ acts an oncogenic factor by increasing tumor cell motility and invasion (5,31-34). Therefore, TGF- $\beta$ and its functional pathways are considered as potential therapeutic targets for glioma. In the Smad-dependent TGF- $\beta$ signaling pathway, there are 3 types of signal-transducing proteins, including Smad2, Smad3 and Smad4. Smad4 is also an indispensable element of the bone morphogenetic protein (BMP)-signaling pathway. The BMP signaling pathway has been shown to promote tumorigenesis in a murine model of high-grade glioma (35). In addition, Smad4 protein is highly expressed in glioma; therefore, Smad4 is a crucial target gene in the treatment of glioma. Based on the inverse correlation between miR-124 with Smad4 expression within glioma cells, we hypothesized that the lower expression of miR-124 promoted Smad4 expression. Recently, $\mathrm{Zu}$ et al identified Smad4 as a novel target of miR-124 in human non-small cell lung cancer (36). In the present study, we confirmed that Smad4 was a novel direct target gene of miR-124 in C6 glioma cells. These results imply that miR-124 simultaneously influences the TGF- $\beta /$ Smad and BMP signaling pathways by attenuating the Smad4 protein level.

Through a Smad-dependent manner, TGF- $\beta$ can activate the Jak-Stat 3 pathway and promote the self-renewal of human GICs (5). Unexpectedly, we found that in C6 cells, the enhanced miR-124 or decreased Smad4 expression did not affect the secreted LIF protein levels, while Stat 3 and p-Stat 3 levels were decreased at the protein level. Furthermore, upon transfection of the C6 cells with siRNAs against Smad4, Stat3 protein was downregulated. We therefore suggest that at least in $\mathrm{C} 6$ cells, TGF- $\beta /$ Smad4 pathway signaling may activate the Jak/Stat3 pathway, possibly by stimulating other activators, such as TNF- $\alpha$, without depending on enhanced LIF expression. However, exactly which activator was involved should be further addressed. Stat3 is a direct target gene of miR-124 (18). Thus, after restoring miR-124 expression, Stat3 protein levels in C6 cells were downregulated in 2 ways. One way was through direct miR-124 binding to the Stat3 mRNA 3'-UTR, which inhibited Stat3 mRNA transcription. Another way was through miR-124-dependent downregulation of Smad4 protein expression. Therefore, in this study, although we did not evaluate whether the miR-124 controls other target genes, e.g., CDK4 and CDK6, which regulate cell cycle progression, it is plausible that miR-124 partially inhibited C6 cell proliferation by downregulating Stat 3 protein expression.

The c-Myc transcription factor is a critical regulator of cell growth, particularly of cell cycle progression from the G1 to
S phase. Hence, c-Myc expression levels are tightly related to cell proliferation (37). In early-stage cancers, including glioma, TGF- $\beta$ suppresses cell proliferation, whereas in later stages, TGF- $\beta$ promotes cancer cell proliferation by enhancing c-Myc gene expression (38). The molecular mechanisms behing these dual roles in carcinogenesis remain unclear. It has been shown that the c-Myc promoter has several potential SBEs, namely TBE1, TBE2 and TIE. During palatal growth, TGF- $\beta 3$ promotes palatal mesenchymal cell proliferation by enhancing Smad4 binding to TBE1 and activating c-Myc gene expression (20). As shown in this study, c-Myc protein expression was similarly downregulated after enhancing miR-124 expression or silencing Smad4 in C6 cells. These results indicated that the downregulation of Smad4 may cause decreased c-Myc protein expression following the ectopic overexpression of miR-124 in C6 cells. Controversially, other researchers have presented data showing that an adenovirus encoding Smad4 suppresses glioma cell proliferation (39). Therefore, if Smad4 directly controls c-Myc expression in glioma cells, the associated mechanisms should be investigated in greater detail. As is well-known, Stat3 is abnormally overexpressed in various types of cancer cells, including glioma cells, which induces c-Myc expression and stimulates cell proliferation $(40,41)$. Accordingly, we suggest that after enhancing the miR-124 level, the protein-expression levels of Smad4 and Stat3 are suppressed and eventually the c-Myc protein level becomes downregulated in C6 cells, representing another novel mechanism for explaining how miR-124 inhibits C6 cell proliferation.

In conclusion, in this study, we found that Smad4 is a novel, direct target of miR-124 in C6 cells and demonstrated that upon miR-124 upregulation Smad4 was downregulated, which may be a major cause of the inhibition of C6 cell proliferation, The results presented in this study confirm that miR-124 may be a potential target in the treatment of glioma, as it effectively inhibits glioma cell proliferation by targeting multiple genes.

\section{Acknowledgements}

The present study was supported by the National Natural Science Foundation of China (no. 81571199) and the Jilin University Youth Science and Technology Innovation Fund (no. 450060507061).

\section{References}

1. Dolecek TA, Propp JM, Stroup NE and Kruchko C: CBTRUS statistical report: Primary brain and central nervous system tumors diagnosed in the United States in 2005-2009. Neuro Oncol 14 (Suppl 5): v1-v49, 2012.

2. Kong LY, Wu AS, Doucette T, Wei J, Priebe W, Fuller GN, Qiao W, Sawaya R, Rao G and Heimberger AB: Intratumoral mediated immunosuppression is prognostic in genetically engineered murine models of glioma and correlates to immunotherapeutic responses. Clin Cancer Res 16: 5722-5733, 2010.

3. Roberts AB and Wakefield LM: The two faces of transforming growth factor beta in carcinogenesis. Proc Natl Acad Sci USA 100: 8621-8623, 2003.

4. Massagué J: TGFbeta in cancer. Cell 134: 215-230, 2008.

5. Peñuelas S, Anido J, Prieto-Sánchez RM, Folch G, Barba I, Cuartas I, García-Dorado D, Poca MA, Sahuquillo J, Baselga J and Seoane J: TGF-beta increases glioma-initiating cell self-renewal through the induction of LIF in human glioblastoma. Cancer Cell 15: 315-327, 2009.

6. ten Dijke P and Hill CS: New insights into TGF-beta-Smad signalling. Trends Biochem Sci 29: 265-273, 2004. 
7. Bartel DP: MicroRNAs: Genomics, biogenesis, mechanism, and function. Cell 116: 281-297, 2004.

8. Krol J, Loedige I and Filipowicz W: The widespread regulation of microRNA biogenesis, function and decay. Nat Rev Genet 11 : 597-610, 2010.

9. Chen CZ: MicroRNAs as oncogenes and tumor suppressors. N Engl J Med 353: 1768-1771, 2005.

10. Bartel DP: MicroRNAs: Target recognition and regulatory functions. Cell 136: 215-233, 2009.

11. Lawler $\mathrm{S}$ and Chiocca EA: Emerging functions of microRNAs in glioblastoma. J Neurooncol 92: 297-306, 2009.

12. Novakova J, Slaby O, Vyzula R and Michalek J: MicroRNA involvement in glioblastoma pathogenesis. Biochem Biophys Res Commun 386: 1-5, 2009.

13. Silber J, James CD and Hodgson JG: microRNAs in gliomas: Small regulators of a big problem. Neuromolecular Med 11: 208-222, 2009

14. Yoo AS, Sun AX, Li L, Shcheglovitov A, Portmann T, Li Y, Lee-Messer C, Dolmetsch RE, Tsien RW and Crabtree GR: MicroRNA-mediated conversion of human fibroblasts to neurons Nature 476: 228-231, 2011.

15. Xia H, Cheung WK, Ng SS, Jiang X, Jiang S, Sze J, Leung GK, Lu G, Chan DT, Bian XW, et al: Loss of brain-enriched miR-124 microRNA enhances stem-like traits and invasiveness of glioma cells. J Biol Chem 287: 9962-9971, 2012.

16. Cao X, Pfaff SL and Gage FH: A functional study of miR-124 in the developing neural tube. Genes Dev 21: 531-536, 2007.

17. Silber J, Lim DA, Petritsch C, Persson AI, Maunakea AK, Yu M, Vandenberg SR, Ginzinger DG, James CD, Costello JF et al: $\mathrm{miR}-124$ and miR-137 inhibit proliferation of glioblastoma multiforme cells and induce differentiation of brain tumor stem cells. BMC Med 6: 14, 2008

18. Wei J, Wang F, Kong LY, Xu S, Doucette T, Ferguson SD, Yang Y, McEnery K, Jethwa K, Gjyshi O, et al: miR-124 inhibits STAT3 signaling to enhance T cell-mediated immune clearance of glioma. Cancer Res 73: 3913-3926, 2013.

19. Schwartz JP and Wilson DJ: Preparation and characterization of type 1 astrocytes cultured from adult rat cortex, cerebellum, and striatum. Glia 5: 75-80, 1992

20. Zhu X, Ozturk F, Liu C, Oakley GG and Nawshad A: Transforming growth factor- $\beta$ activates c-Myc to promote palatal growth. J Cell Biochem 113: 3069-3085, 2012.

21. Furnari FB, Fenton T, Bachoo RM, Mukasa A, Stommel JM, Stegh A, Hahn WC, Ligon KL, Lou is DN, Brennan C, et al: Malignant astrocytic glioma: Genetics, biology, and paths to treatment. Genes Dev 21: 2683-2710, 2007.

22. Vehlow A and Cordes N: Invasion as target for therapy of glioblastoma multiforme. Biochim Biophys Acta 1836: 236-244, 2013.

23. Lv XB, Jiao Y, Qing Y, Hu H, Cui X, Lin T, Song E and Yu F: miR-124 suppresses multiple steps of breast cancer metastasis by targeting a cohort of pro-metastatic genes in vitro. Chin J Cancer 30: 821-830, 2011.

24. Lang Q and Ling C: MiR-124 suppresses cell proliferation in hepatocellular carcinoma by targeting PIK3CA. Biochem Biophys Res Commun 426: 247-52, 2012.

25. Xia J, Wu Z, Yu C, He W, Zheng H, He Y, Jian W, Chen L, Zhang L and $\mathrm{Li} \mathrm{W}$ : miR-124 inhibits cell proliferation in gastric cancer through down-regulation of SPHK1. J Pathol 227: 470-480, 2012.
26. An L, Liu Y, Wu A and Guan Y: microRNA-124 inhibits migration and invasion by down-regulating ROCK1 in glioma. PLoS One 8: e69478, 2013.

27. Deng X, Ma L, Wu M, Zhang G, Jin C, Guo Y and Liu R: miR-124 radiosensitizes human glioma cells by targeting CDK4 J Neurooncol 114: 263-274, 2013.

28. Li KK, Pang JC, Ching AK, Wong CK, Kong X, Wang Y, Zhou L, Chen $\mathrm{Z}$ and $\mathrm{Ng} \mathrm{HK}$ : miR-124 is frequently down-regulated in medulloblastoma and is a negative regulator of SLC16A1. Hum Pathol 40: 1234-1243, 2009.

29. Lu SH, Jiang XJ, Xiao GL, Liu DY and Yuan XR: miR-124a restoration inhibits glioma cell proliferation and invasion by suppressing IQGAP1 and $\beta$-catenin. Oncol Rep 32: 2104-2110, 2014.

30. Lv Z and Yang L: miR-124 inhibits the growth of glioblastoma through the downregulation of SOS1. Mol Med Rep 8: 345-349, 2013.

31. Piek E, Westermark U, Kastemar M, Heldin CH, van Zoelen EJ, Nistér M and Ten Dijke P: Expression of transforming-growthfactor (TGF)-beta receptors and Smad proteins in glioblastoma cell lines with distinct responses to TGF-beta1. Int J Cancer 80: 756-763, 1999.

32. Kjellman C, Olofsson SP, Hansson O, Von Schantz T, Lindvall M, Nilsson I, Salford LG, Sjögren HO and Widegren B: Expression of TGF-beta isoforms, TGF-beta receptors, and SMAD molecules at different stages of human glioma. Int J Cancer 89: 251-258, 2000.

33. Rich JN: The role of transforming growth factor-beta in primary brain tumors. Front Biosci 8: e245-e260, 2003.

34. Golestaneh N and Mishra B: TGF-beta, neuronal stem cells and glioblastoma. Oncogene 24: 5722-5730, 2005.

35. Hover LD, Owens P, Munden AL, Wang J, Chambless LB, Hopkins CR, Hong CC, Moses HL and Abel TW: Bone morphogenetic protein signaling promotes tumorigenesis in a murine model of high-grade glioma. Neuro Oncol 18: 928-938, 2016.

36. Zu L, Xue Y, Wang J, Fu Y, Wang X, Xiao G, Hao M, Sun X, Wang Y, Fu G and Wang J: The feedback loop between miR-124 and TGF- $\beta$ pathway plays a significant role in non-small cell lung cancer metastasis. Carcinogenesis 37: 333-343, 2016.

37. Bretones G, Delgado MD and León J: Myc and cell cycle control. Biochim Biophys Acta 1849: 506-516, 2015.

38. Singh G, Singh SK, König A, Reutlinger K, Nye MD, Adhikary T, Eilers M, Gress TM, Fernandez-Zapico ME and Ellenrieder V: Sequential activation of NFAT and c-Myc transcription factors mediates the TGF-beta switch from a suppressor to a promoter of cancer cell proliferation. J Biol Chem 285: 27241-27250, 2010.

39. Yang Z, Zhong L, Zhong S, Xian R and Yuan B: Adenovirus encoding Smad4 suppresses glioma cell proliferation and increases apoptosis through cell cycle arrest at G1 phase. Int Immunopharmacol 25: 169-173, 2015 .

40. Kiuchi N, Nakajima K, Ichiba M, Fukada T, Narimatsu M, Mizuno K, Hibi M and Hirano T: STAT3 is required for the gp130-mediated full activation of the c-myc gene. J Exp Med 189: 63-73, 1999

41. Lin YM, Wang CM, Jeng JC, Leprince D and Shih HM: HIC1 interacts with and modulates the activity of STAT3. Cell Cycle 12: 2266-2276, 2013 